\title{
TAGLN2 cDNA Cloning from Bufo japonicus formosus and its Diversity Analysis
}

\author{
Hui Zhuge ${ }^{2}$, Xin Gao ${ }^{1,3,4}$, Xianyu Yang ${ }^{1,3,4 *}$ \\ ${ }^{1}$ College of Animal Science and Technology, College of Veterinary Medicine, Zhejiang A\&F University, \\ Hangzhou, 311300, China;
}

${ }^{2}$ Bureau of West Lake Forestry and Water Conservancy of Hangzhou, Hangzhou, 310013, China

${ }^{3}$ Key Laboratory of Applied Technology on Green-Eco-Healthy Animal Husbandry of Zhejiang Province, Hangzhou 311300, China

${ }^{4}$ Zhejiang Provincial Engineering Laboratory for Animal Health Inspection and Internet Technology, Hangzhou 311300, China

*Corresponding Author: Xianyu Yang, College of Animal Science and Technology, College of Veterinary Medicine, Zhejiang A\&F University, Hangzhou 311300, China

\begin{abstract}
During the study of bioactive polypeptides included in Bufo skin, a cDNA segment with partial transgelin-2 (TAGLN2) ORF (open reading frame) was cloned from Japanese toad Bufo japonicus formosus skin cDNA plasmid library (accession number: JX197456). To confirm the expression of the full length TAGLN2, two primers (P1 and P2) were designed based on the partial TAGLN2 sequence, and two PCR (polymerase chain reaction) reactions (P1 and XhoTT, P2 and SP6 as a pair of primers, respectively) were performed to clone the 5'-and 3'-UTR (untranslated region) using the same cDNA library as templates, and the PCR products were cloned. Based on the newly cloned 5'-and 3'-UTR sequences of TAGLN2, two new primers (P3 and P4) were designed and the second round PCR was performed by pairing P3 with XhoTT, and P4 with SP6, respectively. As the result, several TAGLN2 cDNA including full length or partial ORF were cloned indicating the expression of full length TAGLN2 in Japanese toad. Concerning its diversity, one SNP (single nucleotide polymorphism sites) in ORF was found leading to no amino acid change, and different length of 5'-UTR as well as 6 SNP in 3'-UTR was indicated.
\end{abstract}

Keywords: Bufo japonicus formosus; Transgelin-2; cDNA cloning; Diversity

\section{INTRODUCTION}

Chan'pi (skin), Chan'yi (cortex) and Chan'su (secretions) are all Bufo skin-origin materials having been widely used in many prescriptions of traditional Chinese medicine and showing nice effects on pain relief, swelling and tumor control as well as many other diseases ${ }^{[1-4]}$. Cinobufacini, aqueous extract of dried Bufo skin, is such a clinical drug mainly aimed at the advanced cancer ${ }^{[5,6]}$. Recent studies indicated that the polypeptides purified from Cinobufacini injection showed similar antitumor activity as the injection itself in vitro experiment ${ }^{[7]}$. During the analysis of polypeptides included in Bufo skin, a cDNA clone with partial transgelin-2 (TAGLN2) ORF was isolated from Japanese toad skin cDNA plasmid library ${ }^{[8]}$.

TAGLN2, also called SM22 $\beta$ (smooth muscle 22), is an actin binding protein which was purified from chicken gizzard smooth muscle for the first time ${ }^{[9,10]}$ involving in cell proliferation and differentiation ${ }^{[11]}$, and oncogenesis ${ }^{[12,13]}$. TAGLN2 has become a potential marker of tumorigenesis, provides a reference for early diagnosis, treatment and monitoring of tumors ${ }^{[14]}$. To confirm the expression of TAGLN2 with full length ORF, cDNA cloning was carried out from the skin cDNA plasmid library of Japanese toad based on the partial TAGLN2 sequence cloned previously ${ }^{[8]}$.

\section{Materials AND MethodS}

\subsection{Experimental Materials and Reagents}

Japanese toad skin cDNA plasmid library held by the Japan Advanced Industrial Science and Technology (AIST, Tsukuba, Japan) was authorized Zhejiang A\&F University (ZAFU) for research 
as part of a Material Transfer Agreement. Concerning the library, as reported previously ${ }^{[15]}$, whose vector is pSD64TR (3250 base pairs), upstream primer is SP6 and the downstream one is S.D.A., and EcoR I and Xho I are the cloning sites. cDNA length is ranged 500-2 000 base pairs (bp). PCR kit purchased from TaKaRa; pGM-T from Tiangen (Beijing, China); the primer synthesis and DNA sequencing were done by GENEWIZ (Suzhou, China).

\subsection{Primer Design and Cloning of TAGLN2 with Whole ORF from Japanese Toad}

Based on the partial Japanese toad TAGLN2 cDNA (GenBank accession number: JX197456) ${ }^{[8]}$, two primers (P1, P2) were designed (Table 1). P2 was paired with SP6 for the cloning of the $5^{\prime}$-UTR (Group 1), and P1 paired with XhoTT (a self-designed primer complementary with the area compassing the connection point of cDNA poly(A) tail and the downstream cloning site of Xho I) for the cloning of the 3'-UTR (Group 2) (Table 2). Based on the sequences newly cloned 5'-UTR and 3'UTR, two other primers (P3, P4) were designed (Table 1) for the second round PCR. P4 paired with SP6 (Group 3) and P3 paired with XhoTT (Group 4) for the cloning of cDNA with original ORF (Table 2). Japanese toad skin cDNA plasmid library was used as template in the current study. All four PCR constitutions are the same except the primers as indicated in Table 2. PCR parameters are following: $94^{\circ} \mathrm{C} / 3 \mathrm{~min} ;\left(94^{\circ} \mathrm{C} / 30 \mathrm{~s}, 52^{\circ} \mathrm{C} / 30 \mathrm{~s}, 72^{\circ} \mathrm{C} / 2.5 \mathrm{~min}\right) \times 35$ cycles. PCR products were ligated into $\mathrm{pGM}-\mathrm{T}$, and the candidates of positive clones were sequenced.

Table1. Primers used in current study

\begin{tabular}{|c|c|}
\hline Name & Sequences \\
\hline SP6 & 5'-ATTTAGGTGACACTATAGAA-3' \\
\hline XhoTT & 5'-AGATCTCTCGAGTTTTTTTTTTTT-3' \\
\hline P1 & 5'-GCTAAAATCCAGACATC-3' \\
\hline P2 & 5'-GATGAGTGGATGATCTG-3' \\
\hline P3 & 5'-AACCACCAACCACTAAAATGG-3' \\
\hline P4 & 5'-TAAACATAGATTGGTTTTATT-3' \\
\hline
\end{tabular}

Table2. PCR constitutions for TAGLN2 cloning from Japanese Bufo

\begin{tabular}{|c|c|}
\hline Components & Vol $(\mu \mathrm{l})$ \\
\hline $10 x T a q$ buffer & 2.0 \\
\hline $10 \mathrm{mM}$ dNTPs & 0.8 \\
\hline Taq $(5 \mathrm{U} / \mu \mathrm{l})$ & 0.2 \\
\hline Primer $1(2 \mu \mathrm{M})^{*}$ & 1.0 \\
\hline Primer $2(2 \mu \mathrm{M})^{*}$ & 1.0 \\
\hline Template** & 0.5 \\
\hline $\mathrm{H}_{2} \mathrm{O}$ & 14.5 \\
\hline
\end{tabular}

Notes: * SP6 paired with P2, P1 with XhoTT, SP6 with P4 and P3 with XhoTT in Group 1, 2, 3 and 4, respectively. ** Japanese toad skin plasmid cDNA library

\subsection{Homology Analysis of TAGLN2 Amino Acids}

After sequencing, DNAstar/EditSeq was used to find out ORF and deduce the amino acid sequence of the encoding protein, then the amino acid sequence was applied NCBI blast program (http://blast.ncbi. nlm.nih.gov/Blast.cgi). The homolog sequences of 8 other animals were downloaded and aligned by DNAstar/MegAlign, which was also used to construct the phylogenetic tree of TAGLN2.

\section{Results}

\subsection{TAGLN2 Cloning from B. japonicus formosus}

The first round PCR (lane 1 and 2 in Fig.1) was to clone TAGLN2 cDNA with complete 5'- and 3'UTR, and the second round PCR (lane 3 and 4 in Fig.1) was to clone the whole TAGLN2 ORF with either complete 5'-UTR or complete 3'-UTR. Sequencing analysis indicated that 6 different clones were obtained in Group 1, 4 in Group 2, 7 in Group 3, and 6 in Group 4, totally 23 different clones were obtained. For easy description, these clones were designated as TAGLN2-M-N (M: Group No., $\mathrm{N}$ : Clone No. in each group). 


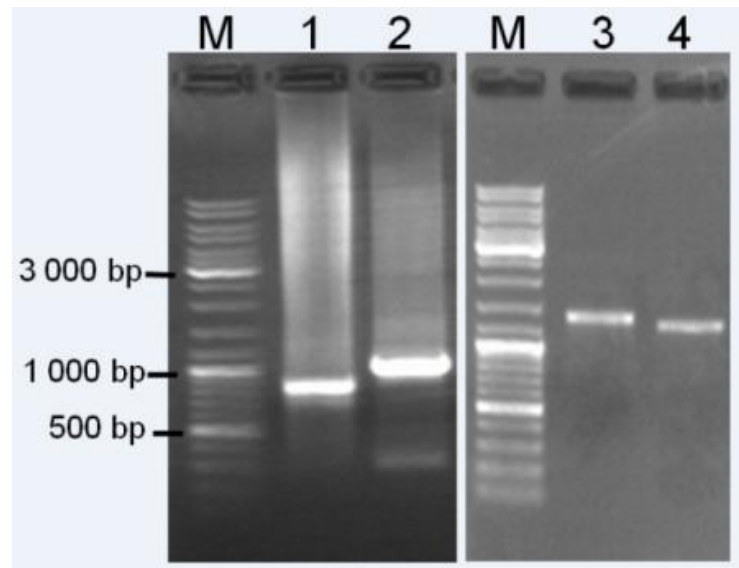

Fig1. PCR products of TAGLN2 amplified from B. japonicus formosus

M: DNA Ladder; 1: SP6 and P2; 2: P1 and XhoTT; 3: SP6 and P4; 4: P3 and XhoTT

\subsection{TAGLN2 cDNA of B. japonicus formosus}

Among the above 23 clones, TAGLN2-3-5 is 1267 bp in length with 86 bp 5'-UTR, 594 bp ORF and 587 bp 3'-UTR encoding a protein consisting of 197 amino acid residues (Fig. 2), whose molecular weight is $21.953 \mathrm{kD}$, closed to that of other animal TAGLN2. Homology analysis of TAGLN2 of Japanese toad indicated 82\% homology with Xenopus laevis (NP_001080783.1), 81\% with Xenopus (Silurana) tropicalis (NP_989354.1), and 71\%-78\% among 6 other animals (Rattus norvegicus, NP_00101314 5.1; Crotalus adamanteus, AFJ51813.1; Mus musculus, NP_848713.1; Homo sapiens, AAH02616.1; Gallus gallus, XP_003643901.1; Danio rerio, NP963870.1) (Fig. 3). So the clone was deposited into GenBank (accession number: KC820703) as TAGLN2 of B. japonicus formosus. The phylogenetic tree is basically consistent with the traditional animal tax onomy (Fig. 4).

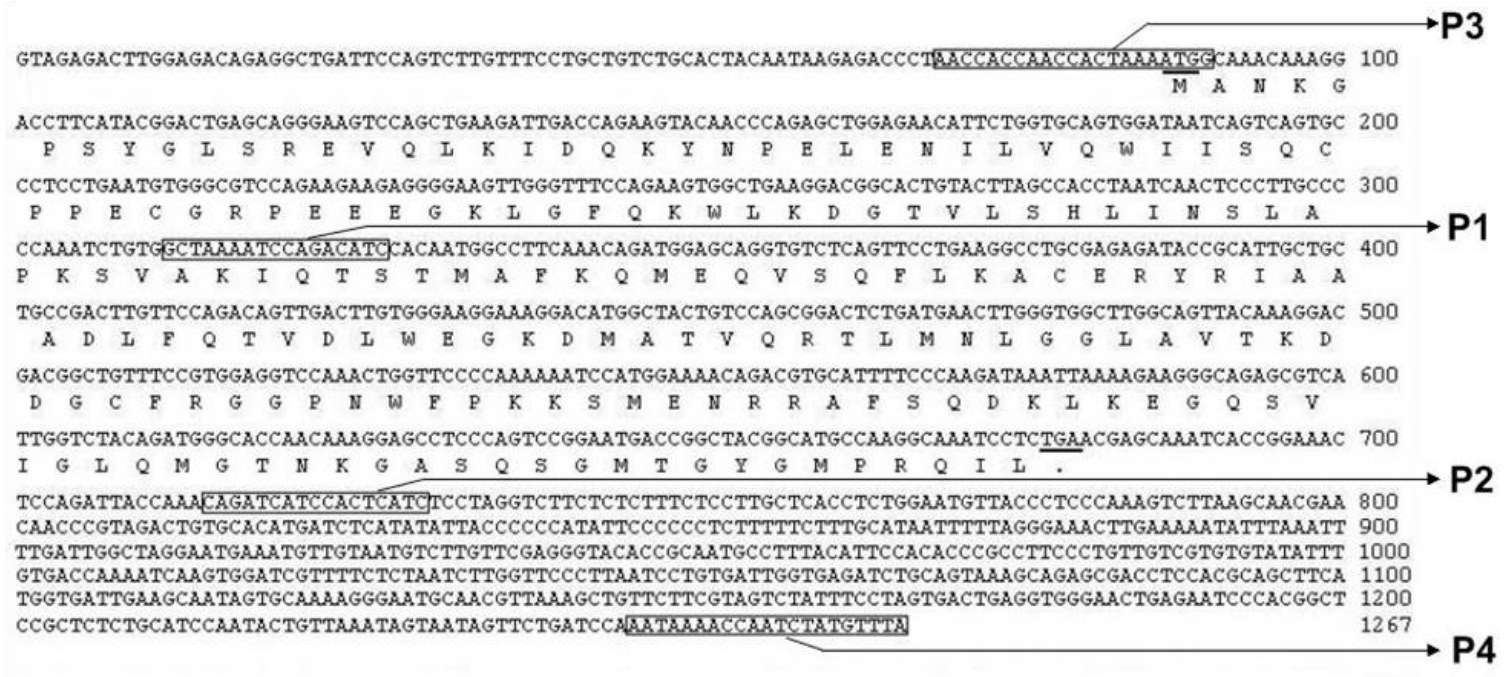

Fig 2. TAGLN2 cDNA of B. japonicus formosus with the whole ORF and the deduced amino acid sequence of the encoding protein

Underline: Start and stop codons; P1-P4: the locations of 4 primers

\subsection{Diversity of TAGLN2 cDNA of B. japonicus formosus}

As describes above, 23 clones were obtained. Due to the use of SP6 as the upstream primer, 5'-UTR sequences of the clones included in Group 1 and 3 should represent the original 5'-UTR in vivo. Meanwhile because of the use of XhoTT as the downstream primer, 3'-UTR sequences of the clones included in Group 2 and 4 should represent original 3'-UTR in vivo. Additionally, because the clones of Group 1, 3 and 4 were derived from the PCR products using SP6 paired with P2/P4, or /XhoTT paired with P3, while P2, P3 and P4, are all located in either 5'-UTR or 3'-UTR as indicated in Fig. 2, therefore, the clones from these 3 groups are supposed to have original ORF in tissue. Here, the TAGLN2 cDNA diversity was analyzed according to ORF, 5'-UTR and 3'-UTR, respectively. 



Fig3. Homology analysis of TAGLN2 of B. japonicus formosus

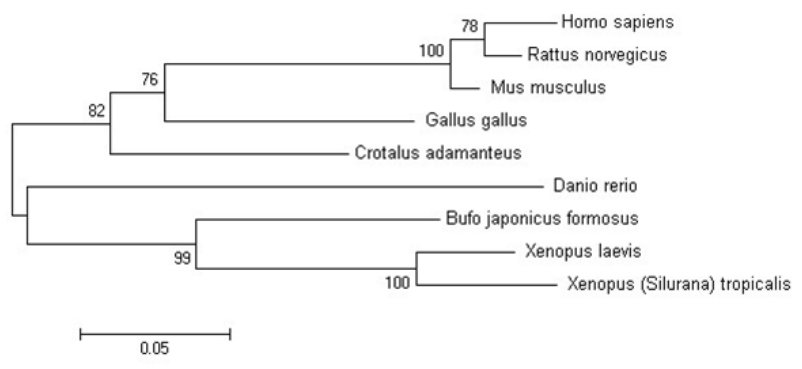

Fig4. Phylogenetic tree of TAGLN2 between B. japonicas formosus and 8 other animals

Concerning ORF, 16 out of 19 clones from Group 1, 3 and 4 have 597 bp ORF with 7 SNP (single nucleotide polymorphism site), the remaining 3 clones have shorter ORF being the same as that reported previously ${ }^{[8]}$. Among 7 SNP, 5 appeared only once, which might be introduced by PCR. However, the remaining 2 are located at the $117^{\text {th }}$ and $564^{\text {th }}$ positions of ORF, which appeared repeatedly in different experiments indicating their objectivity, while they did not lead to amino acid change because of their synonymous mutations (transition between thymine and cytosine). Therefore, all 16 clones encode the same TAGLN2 of Japanese toad.

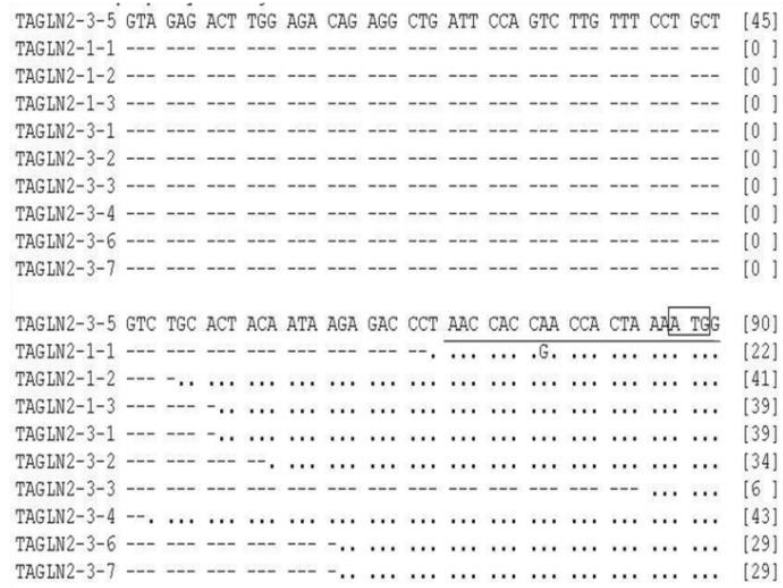

Fig5. Alignment of multiple TAGLN2 5'-UTR sequences of B. japonicus formosus

- : absence of corresponding nucleotide; · : identical nucleotide, Underline: Location of P3 primer; 口: Start codon

With regard to 5'-UTR, 10 clones with full length ORF were analyzed. As shown in Fig. 5, the length is quite different among different clones, the longest one is $86 \mathrm{bp}$ (TAGLN2-3-5), and the shortest one is only 2 bp (TAGLN2-3-3). But the nucleotides of the overlapped area are the same (Fig. 5). About 3'- 
UTR, 11 sequences were compared and 15 SNP appeared, among which 9 appeared only once or in a certain group, most likely introduced by PCR. The remaining 6 were appeared in different experiments indicating their objective existence. Additionally, the length of poly (A) tail was different among clones (Fig. 6).



Fig6. Alignment of multiple TAGLN2 3'-UTR sequences of Bufo japonicus formosus

$-:$ absence of corresponding nucleotide; $::$ identical nucleotide; Underline: Location of P4 primer; $\square$ : Stop codon

\section{DISCUSSION}

Current study clarified the expression of full length TAGLN2 in Japanese toad skin (Fig. 2), and confirmed the existence of partial TAGLN2 expression ${ }^{[8]}$. At the protein level, there is only one amino 
acid difference between TAGLN2 of Japanese Bufo and or Chinese Bufo (B. gargarizans) showing homology as high as $99 \%$. The different amino acid is located in the unimportant area, therefore, both should have the same characteristics as reported in previous study ${ }^{[16]}$. TAGLN2 diversity of Japanese toad is mainly reflected in the length of 5'-UTR (Fig. 5), while ORF and 3'-UTR are relatively conserved (Fig. 6). Homology analysis of Japanese toad TAGLN2 showed 71-82\% with other 8 vertebrates (Fig. 4), indicating its high conservancy in the process of evolution.

As an actin binding protein, TAGLN2 is involved in many physiological and pathological processes by reorganization of cytoskeleton, microfilaments ${ }^{[17-19]}$. Concerning the molecular mechanism of the antitumor activity of TAGLN2, there have been some reports that TAGLN2 noncoding region affects its posttranscriptional translation, and plays a role in inhibiting cancer metastasis together with miRNA ${ }^{[20-22]}$. But, the mechanism is still necessary to be further studied in future.

In fact, cDNA cloning either from Japanese Bufo or Chinese Bufo has been our main study to address the polypeptide ingredients working on tumor control as well as other diseases, and the expression such as Mcl-1, Gal-3, EDF-1, PPDPF and Clu was clarified, all of which are related with oncogenesis or metastasis, and supposed to be the anti-tumor ingredients included in Bufo skin-origin materials ${ }^{[23-}$ ${ }^{27]}$. One more issue is that partial cDNA of $\mathrm{Mcl}-\mathrm{I}^{[23]}, \mathrm{Gal}-3^{[24]}$ has also been cloned. So far, several peptides derived from protein have been reported including Buforin I and II ${ }^{[28]}$, Abhisin ${ }^{[29]}$ and NuBCP-9 ${ }^{[30]}$, either with antimicrobial activity or antitumor activity. Combined all these studies with the report that polypeptides from Cinobufacini has antitumor activity, and the fact that most tradition Chinese medicine is taken orally, it might be not unreasonable to suggest that proteins included in tradition Chinese medicine play their pharmaceutical functions by short polypeptides. The polypeptides included in Chan'pi, Chan'yi and Chan'su, are urgent to be studied.

\section{ACKNOWLEDGEMENT}

This work was partially supported by the grant from National Natural Science Foundation of China (No. 31772409, 31372149).

\section{REFERENCES}

[1] Enomoto A, Rho MC, Komiyama K, et al. Inhibitory effects of bufadienolides on interleukin-6 in MH-60 cells. J Nat Prod, 2004, 67(12): 2070-2072.

[2] Hong Z, Chan K, Yeung H. Simultaneous determination of bufadienolides in the traditional Chinese medicine preparation, Liu-Shen-Wan, by liquid chromatography. J Pharm Pharmacol, 1992, 44(12): 10231026.

[3] Li C, Hashimi SM, Cao S, et al. The mechanisms of Chansu in inducing efficient apoptosis in colon cancer cells. Evid Based Complement Alternat Med, 2013, 2013: 1-11.

[4] Qin TJ, Zhao XH, Yun J, et al. Efficacy and safety of gemcitabine-oxaliplatin combined with huachansu in patients with advanced gallbladder carcinoma. World J Gastroenterol, 2008, 14(33): 5210-5216.

[5] Gomes A, Bhattacharjee P, Mishra R, et al. Anticancer potential of animal venoms and toxins. Indian J Exp Biol, 2010, 48(2): 93-103.

[6] Yin JH, Zhu XY, Shi WD, et al. Huachansu injection inhibits metastasis of pancreatic cancer in mice model of human tumor xenograft. BMC Complement Altern Med, 2014, 14(1): 483.

[7] Wu X, Gao B, Yang J, et al. In vitro anti-proliferation effect of peptides from cinobufacini injection. Acta Pharm Sin, 2012, 47(6): 822-826.

[8] Zhuge H, Yuan J, Zhang S, et al. Cloning and bioinformatic analysis of TAGLN2 cDNA of Bufo japonicus formosus . Acta Pharm Sin, 2013, 48: 250-254.

[9] Lee-Miller JP, Heeley DH, Smillie LB, et al. Isolation and characterization of an abundant and novel 22kDa protein (SM22) from chicken gizzard smooth muscle. J Biol Chem, 1987, 262: 2988-2993.

[10] Lee-Miller JP, Heeley DH, Smillie LB. An abundant and novel protein of $22 \mathrm{kDa}$ (SM22) is widely distributed in smoothmuscles . Biochem J, 1987, 244: 705-709.

[11] Zhang JC, Helmke BP, Shum A, et al. SM22beta encodes a lineage-restricted cytoskeletal protein with a unique developmentally regulated pattern of expression. Mech Dev, 2002, 115: 161-166.

[12] Jonsson G, Naylor TL, Vallon-Christersson J, et al. Distinct genomic profiles in hereditary breast tumors identified by array-based comparative genomic hybridization. Cancer Res, 2005, 65: 7612-7621.

[13] Shi YY, Wang HC, Yin YH, et al. Identification and analysis of tumour-associated antigens in hepatocellular carcinoma. Br J Cancer, 2005, 92: 929-934.

[14] Rho JH, Roehrl M, Wang JY. Tissue proteomics reveals differential and compartment-specific expression 
of the homologs transgelin and transgelin-2 in lung adenocarcinoma and its stroma . J Proteome Res, 2009, 8: $5610-5618$.

[15] Ji Y, Zhuge H, Zhang S, et al. Cloning the sterol carrier protein 2 genes of Japanese toad (Bufo japonicus formosus) and Chinese toad (Bufo gargarizans) and its tissue expression analysis. Zool Res, 2014, 35(5): 398-403.

[16] Zhang S, Zhuge H, Zhang S, et al. Cloning and tissue distribution of TAGLN2 in Bufo gargarizans. Life Sci Res, 2013, 17(6): 481-485. (in Chinese)

[17] Goodman A, Goode BL, Matsudaira P, et al. The Saccharomyces cerevisiae calponin/ transgelein homolog Scp1 functions with fimbrin to regulate stability and organization of the actin cytoskeleton. Mol Biol Cell, 2003, 14: 2617-2629.

[18] Yamazaki D, Kurisu S, Takenawa T. Regulation of cancer cell motility through actin reorganization. Cancer Sci, 2005, 96: 379-386.

[19] Leung WKC, Ching AKK, Chan AWH, et al. A novel interplay between oncogenic PFTK1 protein kinase and tumor suppressor TAGLN2 in the control of liver cancer cell motility [J]. Oncogene, 2011, 30: 44644475.

[20] Wang LH, Liu W, Jiang W, et al. A miRNA binding site single-nucleotide polymorphism in the 3'-UTR region of the IL23R gene is associated with breast cancer. PloS One, 2012, 7: e49823.

[21] Yoshino H, Chiyomaru T, Enokida H, et al. The tumour suppressive function of miR-1 and miR-133a targeting TAGLN2 in bladder cancer. Br J Cancer, 2011, 104: 808-818.

[22] Nohata N, Sone Y, Hanazawa T, et al. miR-1 as a tumor suppressive microRNA targeting TAGLN2 in head and neck squamous cell carcinoma. Oncotarget, 2011, 2: 29-42.

[23] Hu Q, Zhang S, Yang X, et al. Bufo gargarizans mcl-1 cloning and its prokaryotic recombinant protein expression. Acta Pharm Sin, 2013, 48(10): 1624-1628. (In Chinese)

[24] Xu Y, Song M, Yang X, et al. Cloning and sequencing of Bufo gargarizans galectin-3 cDNA and the construction of the vector for its prokaryotic expression. J Southwest Univ, 2012, 34: 46-52. (In Chinese)

[25] Fang Q, Yue J, Yang X. Cloning and its bioinfomatic analysis of EDF1 cDNA from two Bufo species. J China Pharm Univ, 2014, 45(4): 480-485. (In Chinese)

[26] Yue J, Jiang G, Yang X, et al. PPDPF cDNA cloning from two Bufo species and tissue expression in $B$. gargarizans. IJRSB, 2018, 6(4): 35-40.

[27] Jiang G, Yuan J, Lin Y, et al. Cloning and tissue expression of Clusterin in Bufo gargarizans. IJRSB, 2020, Accepted.

[28] Cho JH, Sung BH, Kim SH. Buforins: Histone H2A-derived antimicrobial peptides from toad stomach. Biochim Biophys Acta, 2009, 1788 (8): 1564-1569.

[29] De Zoysa M, Nikapitiya C, Whang I, et al. Abhisin: a potential antimicrobial peptide derived from histone H2A of disk abalone (Haliotis discus discus). Fish Shellfish Immunol, 2009, 27(5):639-46.

[30] Kolluri SK, Zhu X, Zhou X, et al. A short Nur77-derived peptide converts Bcl-2 from a protector to a killer. Cancer Cell, 2008, 14(4): 285-98.

Citation: Xianyu Yang, et.al, "TAGLN2 cDNA Cloning from Bufo japonicus formosus and its Diversity Analysis", International Journal of Research Studies in Biosciences, 8(4), pp. 1-7. DOI: http://dx.doi.org/10.20431/2349-0365.0804001

Copyright: (C) 2020 Authors, This is an open-access article distributed under the terms of the Creative Commons Attribution License, which permits unrestricted use, distribution, and reproduction in any medium, provided the original author and source are credited. 